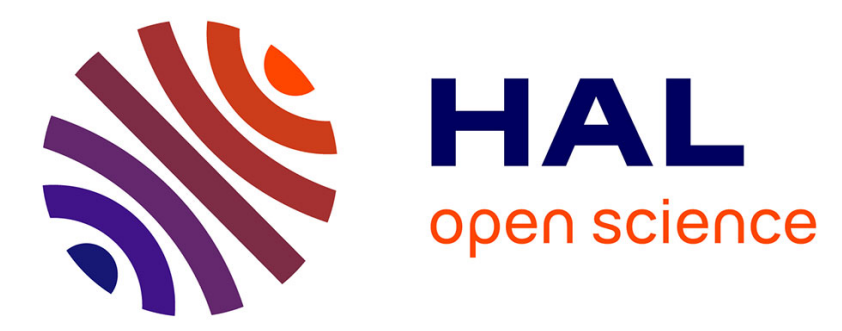

\title{
Three-dimensional temperature imaging around a gold microwire
}

\author{
Pierre Bon, Nadia Belaid, Denis Lagrange, Christian Bergaud, Hervé \\ Rigneault, Serge Monneret, Guillaume Baffou
}

\section{- To cite this version:}

Pierre Bon, Nadia Belaid, Denis Lagrange, Christian Bergaud, Hervé Rigneault, et al.. Threedimensional temperature imaging around a gold microwire. Applied Physics Letters, 2013, 102, pp.244103. 10.1063/1.4811557 . hal-00904675

\section{HAL Id: hal-00904675 \\ https://hal.science/hal-00904675}

Submitted on 15 Nov 2013

HAL is a multi-disciplinary open access archive for the deposit and dissemination of scientific research documents, whether they are published or not. The documents may come from teaching and research institutions in France or abroad, or from public or private research centers.
L'archive ouverte pluridisciplinaire HAL, est destinée au dépôt et à la diffusion de documents scientifiques de niveau recherche, publiés ou non, émanant des établissements d'enseignement et de recherche français ou étrangers, des laboratoires publics ou privés. 


\title{
Three-dimensional temperature imaging around a gold microwire
}

\author{
Pierre Bon ${ }^{1}$, Nadia Belaid ${ }^{2,3}$, Denis Lagrange ${ }^{2,3}$, Christian Bergaud ${ }^{2,3}$, \\ Hervé Rigneault ${ }^{1}$, Serge Monneret ${ }^{1}$, and Guillaume Baffou ${ }^{1 *}$ \\ ${ }^{1}$ Institut Fresnel, CNRS, Aix Marseille Université, \\ Centrale Marseille, UMR 7249, 13013 Marseille, France \\ ${ }^{2}$ CNRS, LAAS, 7 avenue du Colonel Roche, F-31400 Toulouse, France and \\ ${ }^{3}$ Université de Toulouse, LAAS, F-31400 Toulouse, France
}

(Dated: May 21, 2013)

\begin{abstract}
We report on the temperature mapping around a resistively heated gold microwire. The temperature is determined by measuring the thermal-induced distortion of an incident optical wavefront crossing the system. The optical technique we introduce herein allows, in addition to 3-dimensional temperature measurements, a retrieval of the heat source density at optical resolution. Experimental results are supported by finite element simulations and electric measurements. Applications are envisioned in microelectronics, microfluidics or nanochemistry.
\end{abstract}

Keywords: Thermal microscopy, wavefront sensing, microelectronics

*guillaume.baffou@fresnel.fr 
The study of resistively heated nano- and micro-systems, such as metal microwires and carbon nanotubes, has received much interest during these last few years. This area of research benefited from recent developments of new fabrication processes in nanotechnologies and is motivated by applications in microelectronics and chemical/gas sensing. In microelectronics, what currently limits the downscaling is heat dissipation, which has to remain below $100 \mathrm{~W} / \mathrm{cm}^{2}$ in silicon chips and this limit was reached in $2003 .{ }^{1}$ Hence, the development of lower consumption technologies becomes now vital in order to achieve higher transistor density on chip. The other motivation concerns the development of nanoscale chemical sensors based on one-dimensional nanostructures (nanotubes, microwires, nanofibers, etc). ${ }^{2-4}$ The presence of gas or chemicals around the nano-object changes its electrical resistance by adsorption, with the magnitude of change directly related to the gas concentration. This effect usually operates at high temperature. A proper choice of heater voltage is critical in determining the response characteristics of the sensor.

However, these areas of research suffer from the lack of efficient thermal microscopy techniques. The actual temperature of the system is usually not determined accurately, which is yet a crucial parameter. Some microscopy techniques have been developed recently but they are usually slow, invasive or suffer from a low spatial resolution. ${ }^{5-11}$

We have recently developed a temperature microscopy technique, named TIQSI (for Temperature imaging using quadriwave lateral shearing interferometry), based on far-field optical measurements. ${ }^{12}$ This technique is based on measurements of thermal-induced variation of the surroundings refractive index using a wavefront sensor. In a previous work, we illustrated the capabilities of the TIQSI technique for applications in nanoplasmonics by measuring the temperature and the absorption cross section of gold nanoparticles heated by light absorption. ${ }^{13}$

In this article, we show how the TIQSI technique can be used as well to investigate resistively heated systems. Experimental measurements were conducted on a gold microwire lying on a silicon substrate. We explain how both the temperature, the heat power density along the microwire and the total delivered power can be retrieved quantitatively. Furthermore, we explain how the TIQSI technique can be upgraded to map the temperature distribution in three dimensions using a semi-empirical procedure. Finally, we explain how the TIQSI retrieval algorithm has to be updated when the source of heat is embedded in a multilayer system featuring various thermal conductivities. Experimental data are sup- 
ported by finite-element numerical simulations.

We chose to conduct all the experiments on a gold microwire, $40 \mathrm{~nm}$ thick, $1 \mathrm{\mu m}$ large and $80 \mu \mathrm{m}$ long (Fig.1). The extremities of the wire were connected to two gold electrodes and the circuit was electrically isolated from the underlying silicon substrate by a $210 \mathrm{~nm}$ thick $\mathrm{SiO}_{2}$ layer, and passivated by a $100 \mathrm{~nm}$ thick $\mathrm{SiO}_{2}$ layer deposited on top. The fabrication process is described in supplementary notes. ${ }^{14}$ Finally, a water drop was deposited on top of the sample just before the measurements. To perform the temperature measurement, the sample was mounted on a reflection optical microscope (as silicon is not transparent) equipped with a water-dipping objective (Nikon $\times 60, N A=1.0)$ immersed in the water drop. A Köhler configuration was used to illuminate the sample with a collimated beam.

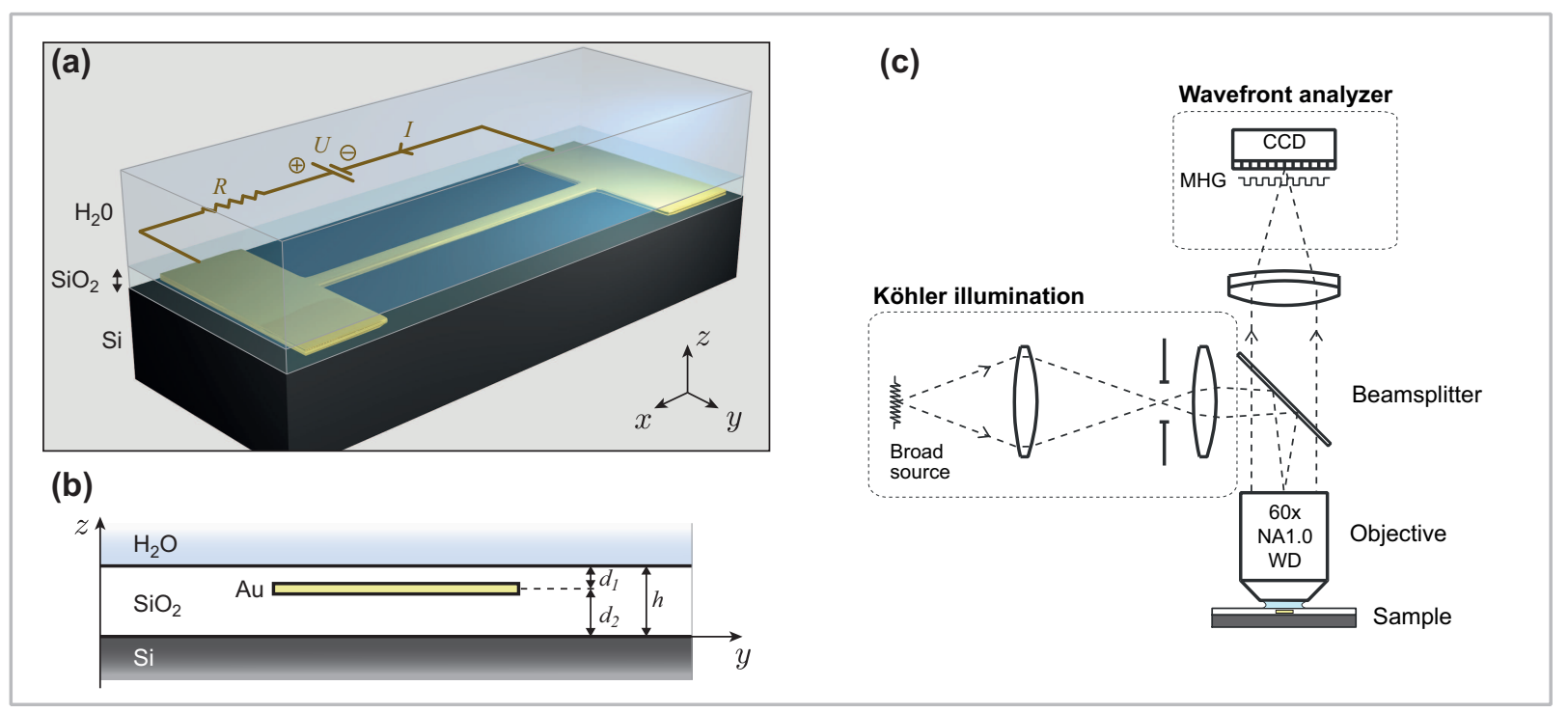

FIG. 1. a) Schematic of the electric circuit. A gold microwire connected to two gold electrodes is embedded within a $\mathrm{SiO}_{2}$ layer. b) Side view of the microwire and the three-layer environment. The microwire is $80 \mu \mathrm{m}$ long et $1 \mu \mathrm{m}$ large. $d_{1}=100 \mathrm{~nm}, d_{2}=210 \mathrm{~nm}, h=d_{1}+d_{2}$. c) Schematic of the optical setup. A wavefront analyzer, consisting of a modified Hartmann Grating (MHG) in front of a CCD sensor, is used to map the thermal induced distortion of the Köhler illumination wavefront.

When a bias is applied to the microwire, heat is generated due to Joule effect, creating a temperature gradient around the microwire and, in particular, in the water layer. The thermal-induced variation of the refractive index of the water layer generates a distortion of this incident wavefront, just like an optical lens or a mirage effect. This distortion is imaged 
using a commercial quadriwave lateral shearing interferometer ${ }^{15}$ used as a wavefront sensor (SID4-HR, Phasics, Palaiseau, France). In practice, two wavefront images are acquired and subtracted: one image while heating and one image without heating. This way, we obtain the optical path difference (OPD) distribution that is specifically due to temperature increase. The spectrum of the illumination was reduced to $450 \pm 20 \mathrm{~nm}$ in order to have approximately the same reflectivity for both gold and silicon $(\approx 40 \%)$ and also a good lateral resolution of $450 \mathrm{~nm}$ (Abbe criterion with spatially coherent illumination). All the experiments were conducted at a controlled ambient temperature of $23^{\circ} \mathrm{C}$.

Once the OPD is measured, one can retrieve the heat source density (HSD in $\mathrm{W} / \mathrm{m}^{2}$ ) and the temperature distributions using a numerical algorithm based on the use of two Green's functions: the thermal Green's function $G_{T}(\mathbf{r})$ (i.e. the temperature distribution generated by a point-like source of heat), and the OPD Green's function $G_{\ell}(\mathbf{r})$ (i.e. the OPD distribution generated by a point-like source of heat). In a previous work, this numerical procedure is explained ${ }^{12}$ in the case of Green's functions associated to a uniform and isotropic medium:

$$
\begin{aligned}
G_{T}(\mathbf{r}) & =1 / r \\
G_{\ell}(\mathbf{r}) & =-\ln (r)+\text { const. }
\end{aligned}
$$

where $r=|\mathbf{r}|$ is the radial coordinate. In the present work, these expressions are no longer valid since the surrounding medium is no longer uniform and composed of three different media ( $\mathrm{Si}, \mathrm{SiO}_{2}$ and water). Hence, one has to use Green's functions related to a 3-layer environment with flat interfaces. This is our approach in this article, and we provide in supplementary notes ${ }^{14}$ all the Green's functions in the cases of one, two and three media separated by flat interfaces.

The overall procedure to retrieve the HSD and the three-dimensional (3D) temperature distributions is the following (see Fig.2): We first de-convolute the measured OPD $\delta \ell$ by the OPD Green's function in order to retrieve the HSD. Then, we convolute this HSD image with the 3D temperature Green's function in order to retrieve the 3D temperature distribution. Such an approach is consistent since a measured OPD image bijectively corresponds to a unique $3 \mathrm{D}$ temperature variation field $\Delta T$, as far as the heat source distribution is $2 \mathrm{D}$. The following equation summarizes the numerical procedure:

$$
\Delta T=G_{T} \otimes\left[G_{\ell}^{-1} \otimes \delta \ell\right]
$$




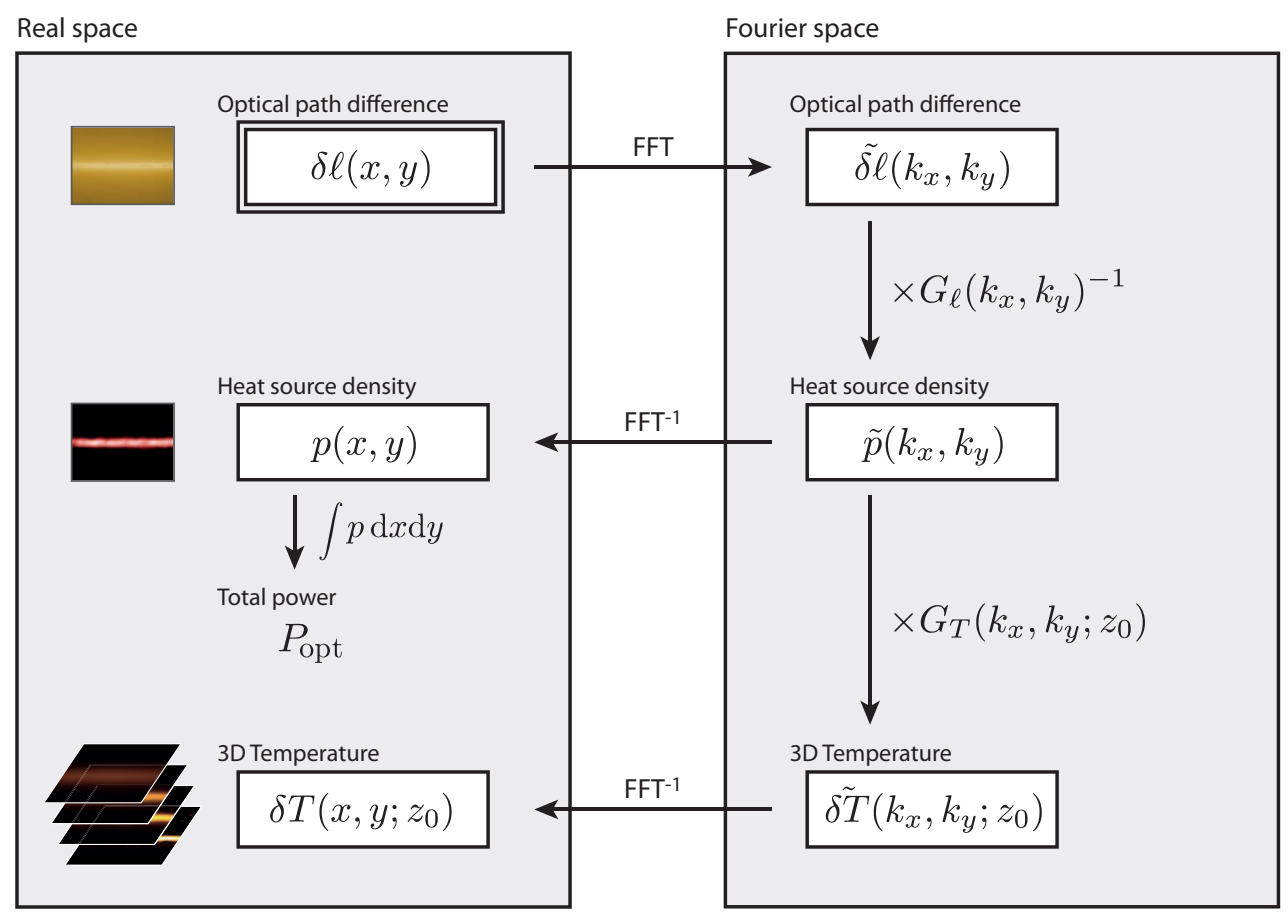

FIG. 2. Schematic of the numerical procedure used to retrieve the heat source density and the 3D temperature distributions from the measured optical path difference. Fourier transforms are used in order to turn convolutions by Green's functions into simple multiplications.

The measurements presented in Fig.3 were performed on the gold microwire heated with a current of $25 \mathrm{~mA}$. The measured OPD distribution is presented in Fig.3(a) and the HSD in Fig.3(b). As expected, the shape of the HSD matches to the microwire morphology. Then, temperature distributions at various heights have been retrieved using the above-mentioned procedure as shown in Fig.3(c). At the microwire level $(z=0)$, the temperature distribution is fairly confined at the vicinity of the wire. ${ }^{8}$ This is due to the high conductivity of the underlying silicon substrate. However, when looking further above the wire, the temperature distribution is naturally broader and weaker.

Our optical approach makes it possible to retrieve the total dissipated heat power $P_{\text {opt }}$ by integrating the HSD distribution. In the experiment reported in Fig.3, we found $P_{\mathrm{opt}}=55$ $\mathrm{mW}$. This value matches well the electric power $P_{\text {elec }}=(U-R I) I=56.4 \mathrm{~mW}$ injected in the system. Such comparisons have been performed for various currents $I$ and they all gave a good agreement between the estimated total dissipated powers, as represented by the linear behavior in Fig.4(a). 


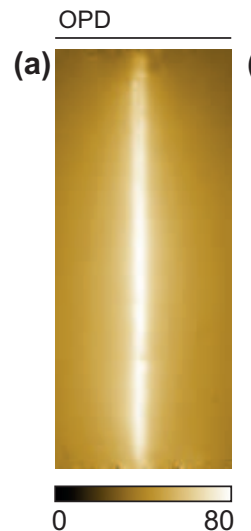

$\delta \ell(\mathrm{nm})$

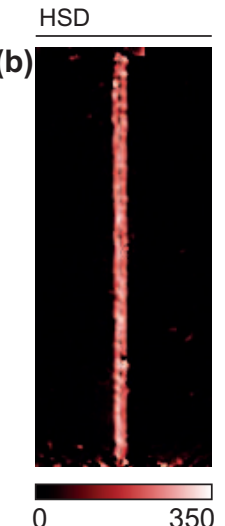

$p\left(\mu \mathrm{W} / \mu \mathrm{m}^{2}\right)$
$\Delta T\left({ }^{\circ} \mathrm{C}\right)$
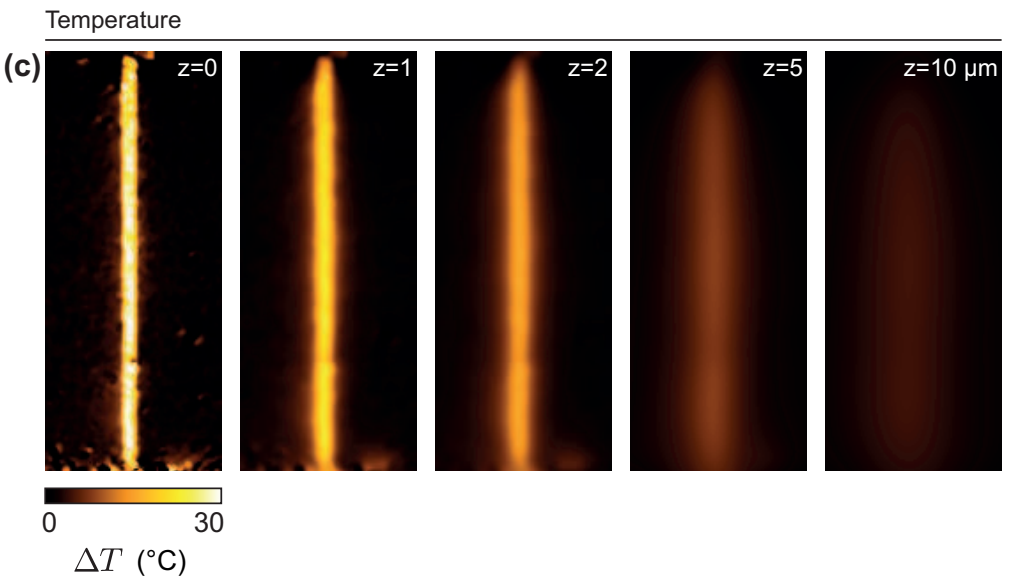

FIG. 3. a) Optical path difference (OPD) measured with the wavefront analyzer due to thermalinduced variation of the surroundings refractive index. b) Heat source density (HSD) retrieved from the OPD. c) Temperature retrieved from the HSD at various heights above the microwire located at $z=0$.

In order to further support the measurements, we also carried out finite-element numerical simulations of the temperature using COMSOL Multiphysics. Details on the simulation parameters are given in supplementary notes. ${ }^{14}$ In Fig.4(f), a simulated temperature profile in the $x z$ plane, crossing the middle of the wire, is compared to the one from the experimental measurements using the algorithm depicted in Fig.2. On the experimental image, we observe a broader and weaker temperature distribution. This observation illustrates the limitation of our optical approach: it is not possible to go below the diffraction limit. However, even if the temperature profile is smoothed due to a limited spatial resolution, let us note that the estimated heat power can still be properly estimated, as observed in Fig.4(a). For more complex systems where the heat power cannot be electrically measured and where the geometry cannot be easily modeled suing COMSOL, our approach appears as a convenient way to unravel the heat delivery patterns.

Let us discuss a final supporting experiment. The resistance $R_{\mathrm{w}}$ of the microwire is a physical quantity that depends on its temperature. Hence, measuring $R_{\mathrm{w}}$ during the experiment stands for a alternative mean to access the microwire temperature $T_{\mathrm{w}}$, as far as the calibration curve is known. A calibration was previously measured by heating the electric circuit in a oven at various temperatures while measuring the resistance of the system. We 
(a)

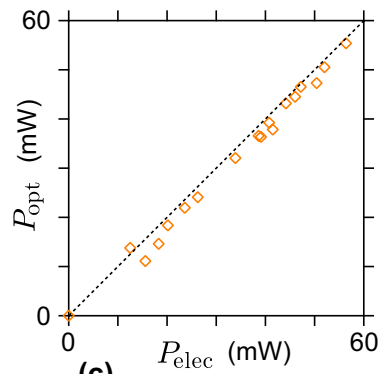

(c)

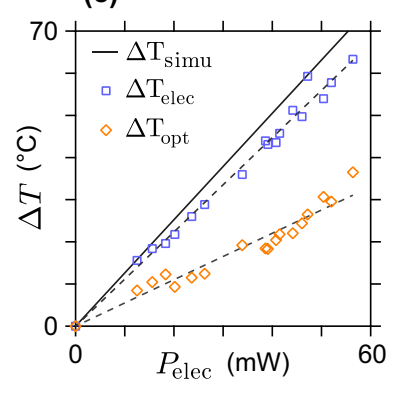

(b)

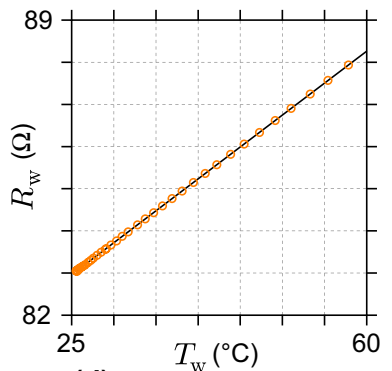

(d)

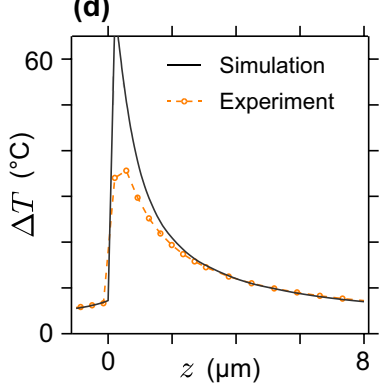

(e)

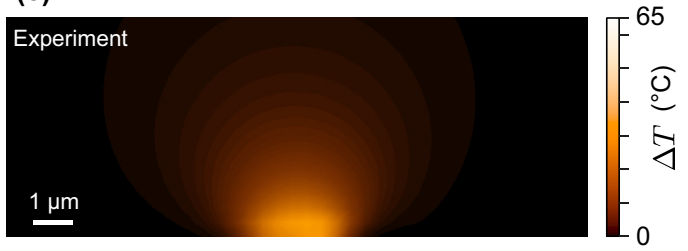

(f)

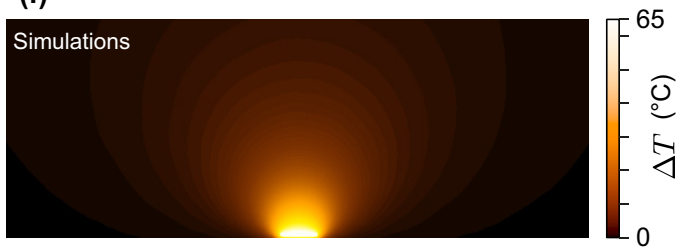

FIG. 4. a) Delivered heat power $P_{\text {opt }}$ estimated using the TIQSI technique compared with the delivered heat power $P_{\text {elec }}=(U-R I) I$ measured electrically. b) Calibration curve relating the microwire resistance and its temperature. c) Estimated temperature using three different approaches: resistance measurements (blue squares), TIQSI measurements (orange diamonds) and finite-element simulations (solid black line). d) Temperature profile along the $z$ axis simulated using COMSOL (solid black line) and retrieved from the experimental measurements using the TIQSI algorithm (dashed orange line). e) Temperature distribution in the $(x, z)$ plane obtained using the TIQSI technique. f) Theoretical temperature distribution in the $(x, z)$ plane modeled using finite-element simulations

found a linear dependence between $R_{\mathrm{w}}$ and $T_{\mathrm{w}}$ as shown in Fig.4(b). Using this calibration curve, we estimated the temperature of the microwire $T_{\text {elec }}$ in parallel to the optical experiments. Figure 4(c) presents a comparison between this resistivity method, finite-element simulations and TIQSI measurements, demonstrating coherent results. A good agreement is observed between the simulations and the resistivity method, while the temperature of the microwire is underestimated due to the diffraction limit in our experiments, as explained above.

In summary, we show how quadriwave lateral shearing interferometry can be used to simply map the temperature in three dimensions around resistively heated systems. We also explain how the heat source density and the actual total heat power delivered by the sys- 
tem can be quantitatively retrieved from the optical measurements. Interestingly, while the temperature may be underestimated when the heat source size is below the diffraction limit, the estimated delivered heat power is on the contrary not affected by any lack of resolution. The simplicity of the experimental procedure and its fast readout rate (around one second to acquire an image) are valuable key points for the characterization of heat release and the investigation of thermal-induced phenomena on the microscale. This readout rate is not a fundamental limitation of the technique, and it could be improved with a faster and better cooled CCD camera. This experimental technique could have valuable applications in microelectronics where heat generation is what currently limits the downscaling of the chips, in microwire gas and chemical sensing, in microfluidics ${ }^{16}$ or in thermal-assisted nanochemistry in solution.

[1] E. Pop, Nano Res 3, 147 (2012).

[2] F. Hernandez-Ramirez, J. D. Prades, R. Jimenez-Diaz, T. Fischer, A. Romano-Rodriguez, S. Mathurd, and J. R. Morante, Phys. Chem. Chem. Phys. 11, 7105 (2009).

[3] A. Balandin, Nature Materials 10, 569 (2013).

[4] S. Ghosh, I. Calizo, D. Teweldebrhan, E. P. Pokatilov, D. L. Nika, A. A. Baladin, W. Bao, F. Miao, and C. N. Lau, Appl. Phys. Lett. 92, 151911 (2008).

[5] C. D. S. Brites, N. J. O. Lima, P. P. Sivlia, A. Millán, V. S. Amaral, F. Palacio, and L. D. Carlos, Nanoscale 4, 4799 (2012).

[6] E. Saïdi, N. Babinet, L. Lalouat, J. Lesueur, L. Aigouy, S. Volz, J. Labéguerie-Eguéa, and M. M., Small 7, 259 (2011).

[7] L. Aigouy, G. Tessier, M. Mortier, and B. Charlot, Appl. Phys. Lett. 87, 184105 (2005).

[8] P. Löw, B. Kim, N. Takama, and C. Bergaud, Small 4, 908 (2008).

[9] B. Samson, L. Aigouy, P. Löw, C. Bergaud, B. J. Kim, and M. Mortier, Appl. Phys. Lett. 92, $023101(2008)$.

[10] L. Aigouy, E. Saïdi, L. Lalouat, J. Labéguerie-Eguéa, M. Mortier, P. Löw, and C. Bergaud, J. Appl. Phys. 106, 074301 (2009).

[11] B. Samson, L. Aigouy, R. Latempa, and G. Tessier, J. Appl. Phys. 102, 024305 (2007).

[12] G. Baffou, P. Bon, J. Savatier, J. Polleux, M. Zhu, M. Merlin, H. Rigneault, and S. Monneret, 
ACS Nano 6, 2452 (2012).

[13] P. Berto, E. Bermúdez Ureña, P. Bon, R. Quidant, H. Rigneault, and G. Baffou, Phys. Rev. B 86, 165417 (2012).

[14] See supplementary material at [URL] for the Green's functions derivation, details on the Comsol simulations and fabrication process.

[15] P. Bon, G. Maucort, B. Wattellier, and S. Monneret, Opt. Express 17, 13080 (2009).

[16] J. Donner, G. Baffou, D. McCloskey, and R. Quidant, ACS Nano 5, 5457 (2011). 